\title{
GALACTIC WORMS
}

\author{
B.-C. KOO, C. HEILES, AND W. T. REACH \\ Astronomy Department, University of California, \\ Berkeley, CA 94720
}

\begin{abstract}
We have found and cataloged over 100 vertical structures in $\mathrm{H} \mathrm{I}$, infrared, and radio continuum emission. These correspond to the $\mathrm{H} \mathrm{I}$ worms detected by Heiles (1984). The infrared and the radio continuum properties of worms suggest that some worms have associated ionized gas. The area filling factor of superbubbles in the inner Galaxy is estimated to be greater than $\sim 0.1$.
\end{abstract}

\section{INTRODUCTION}

Heiles (1984), in his pioneering study of H I shells and supershells, found interesting features in the inner Galaxy, 'H I worms'. By applying a median filter, which enhances small scale structure, to the $\mathrm{H}$ I channel maps near the Galactic plane $\left(|b|<10^{\circ}\right)$, he found that the filtered structure appears to be random in the outer Galaxy, while in the inner Galaxy it tends to run perpendicular to the Galactic plane and individual features tend to persist over a large velocity interval. $\mathrm{He}$ interpreted these vertical structures, or $\mathrm{H}$ I worms, as the walls surrounding superbubbles, which have broken through the thin gaseous disk inside the solar circle.

In this paper we report a systematically determined catalog of 118 similar structures, not only in $\mathrm{H} \mathrm{I}$ but also in the infrared and the radio continuum, and present some preliminary results.

\section{OBSERVATIONS AND DATA ANALYSIS}

The H I 21 -cm maps between $10 \leq l \leq 350$ are made from the Galactic plane survey of Weaver and Williams (1973) and of Kerr et al. (1986). The missing data near the Galactic center were obtained using the Hat Creek 85-foot. The infrared 60 and $100 \mu \mathrm{m}$ maps are made from the IRAS Zodiacal Observation History File (1988). The radio continuum maps are made from the $408 \mathrm{MHz}$ survey of Haslam et al. (1982). All the maps have $0.0^{\circ} 5 \times 0 .^{\circ} 5$ pixel size, and a median filter of $3 .^{\circ} 5 \times 3 .^{\circ} 5$ was applied to see small scale structure near the Galactic plane $\left(|b|<10^{\circ}\right)$. 


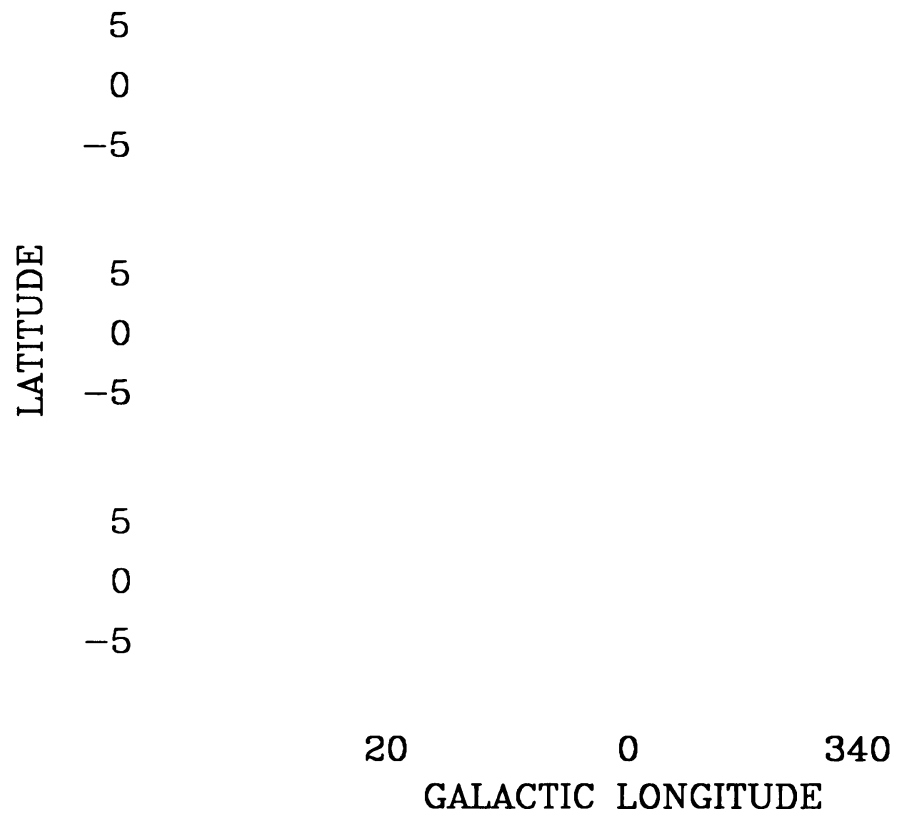

Figure 1. The median-filtered H I, $100 \mu \mathrm{m}$, and $408 \mathrm{MHz}$ map (from top to bottom) in the inner Galaxy.

Figure 1 shows the median-filtered H I map integrated from -200 to +200 $\mathrm{km} \mathrm{s}^{-1}$ in the inner Galaxy together with the corresponding $100 \mu \mathrm{m}$ and $408 \mathrm{MHz}$ maps as an example. The correlation between the $\mathrm{H} \mathrm{I}$ and the $100 \mu \mathrm{m}$ map is remarkable, which proves that most of these structures are real. By cross-correlating the 60 and $100 \mu \mathrm{m}$ maps with the $\mathrm{H}$ I map, but excluding the very central region of the plane $\left(|b|<1^{\circ}\right)$, we found a total of 118 isolated structures that are larger than 5 pixels and appear in both infrared and H I maps. The identification and the cross correlation of structures were made by 'unbiased' search technique based on a computer algorithm which will be described in a subsequent publication. We will call all 118 structures 'worms' even though some of them are small and at high latitudes, so that they are not worms in the sense that they do not 'crawl out of the Galactic plane'.

Not all the worms appear in $408 \mathrm{MHz}$ emission. Among 118 worms, 35 have counterparts at $408 \mathrm{MHz}$. 


\section{INFRARED AND RADIO CONTINUUM PROPERTIES OF WORMS}

Figure 2a shows the median value of the 60 to $100 \mu \mathrm{m}$ intensity ratio, $I_{60} / I_{100}$, versus the median value of the $100 \mu \mathrm{m}$ emissivity, $I_{100} / N_{\mathrm{H} \mathrm{I}}$, of each worm (open circles). The ratio $I_{60} / I_{100}$ is almost independent of $I_{100} / N_{\mathrm{H} \mathrm{I}}$, although there may be a trend such that a worm with a larger $I_{100} / N_{\mathrm{HI}}$ has a larger $I_{60} / I_{100}$. This result is in good agreement with theoretical calculations of Draine and Anderson (1985). The average $I_{60} / I_{100}$ of all worms is large $0.28 \pm 0.06$, and implies a grain size distribution with a relatively large number of smaller grains, which may be due to the population of shock-processed grains.

The large variation of $I_{100} / N_{\mathrm{H}}$ is due to at least three factors: (1) the general increase of the diffuse interstellar radiation field toward the Galactic interior, (2) the worms with associated H II regions, e.g., GW6.5-3.7 (Galactic Worm centered at $l \approx 6.5$ and $b \approx-3.7$ ) with S25, GW14.9-1.6 with S45, GW17.8+3.0 with S54, and G31.6-5.9 with W43 in Figure 1, and (3) the worms with associated molecular gas, e.g., GW1.9+6.0 and GW19.5-6.4. The decomposition of the infrared emissivity into each contribution needs to be done for individual worm.

Figure $2 \mathrm{~b}$ shows the average $408 \mathrm{MHz}$ brightness temperature versus the average $60 \mu \mathrm{m}$ intensity of each worm. There is a good correlation between the two. We are currently unsure of whether the $408 \mathrm{MHz}$ continuum is thermal or nonthermal. One known example of nonthermal emission associated with $\mathrm{H} \mathrm{I}$ is the North Polar Spur (Heiles et al. 1980), and a known example of thermal emission is GW17.8+3.0 (Müller, Reif, and Reich 1987). For the worms with associated H II regions, the $408 \mathrm{MHz}$ emission is likely to be thermal. In Figure $2 \mathrm{~b}$, the straight line is an expected relationship for a diffuse H II region by assuming that all the Lyman $\alpha$ photons are converted to infrared photons. The apparent deficit of $60 \mu \mathrm{m}$ emission possibly arises because some Ly $\alpha$ photons leak through the vertical direction. On the other hand, it may suggest that the $408 \mathrm{MHz}$ emission is non-thermal.

\section{SUMMARY AND DISCUSSION}

We have found that there is a very good correlation between $\mathrm{H} \mathrm{I}$ worms and infrared worms, and that many prominent worms have their counterparts at 408 $\mathrm{MHz}$. We also found that some worms are very likely to have associated ionized gas, which needs to be confirmed by some direct observations, e.g., radio recombination lines.

One primary goal of this work is to answer the following question: "What is the filling factor of superbubbles in our Galaxy ?" We can make a very crude estimate based on our results. A superbubble that has broken through the thin gaseous disk occupies an area with radius comparable to or larger than the scale height $\sim 190 \mathrm{pc}$. In the first and the fourth quadrant, there are about 30 worms that actually rise from the Galactic plane. If we assume that most worms within $4 \mathrm{kpc}$ are detected, then the filling factor of the inner Galaxy is greater than $\sim 0.1$.

A superbubble that has broken through the thin gaseous disk does not necessarily 

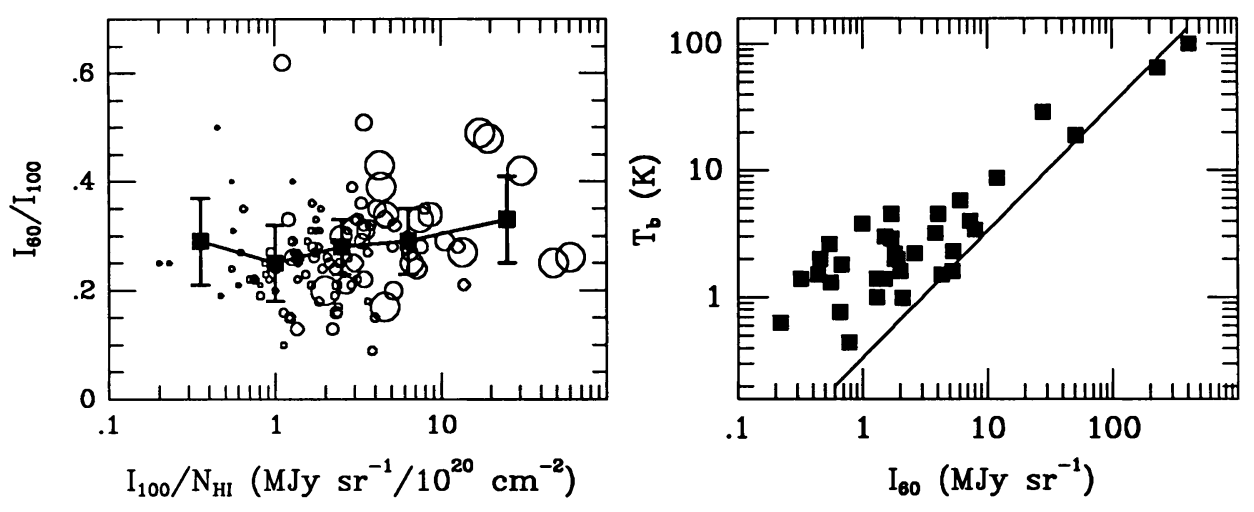

Figure 2. (a) The median value of $I_{60} / I_{100}$ vs. the median value of $I_{100} / N_{\mathrm{H} \text { I }}$ of each worm (open circles). The area of the circle is proportional to the average $100 \mu \mathrm{m}$ intensity. The average values per unit logarithmic interval are shown as squares with $1 \sigma$ error bars. (b) The average $408 \mathrm{MHz}$ brightness temperature vs. the average $60 \mu \mathrm{m}$ intensity of each worm. The straight line is an expected relationship for a diffuse $\mathrm{H}$ II region.

inject hot gas into the halo, because there is a thick $\mathrm{H} I$ layer, and also a even thicker layer of ionized gas (Lockman 1984; Reynolds 1989). However, the cold fragments, which result from the Rayleigh-Taylor instability when the supershell accelerates, are likely to be injected into the halo. According to Mac Low et al. (1989), about $5 \%$ of the shell mass is in cold fragments, then, if we take $50 \mathrm{Myr}$ as the characteristic lifetime of a superbubble, the filling factor of $\sim 0.1$ implies a mass injection rate of $\sim 0.2 M_{\odot} / \mathrm{yr}$.

\section{REFERENCES}

Draine, B.T., and Anderson, N. (1985) Ap. J. 292, 494

Haslam, C.G.T., Salter, C.J., Stofell, H., and Wilson, W.E. (1982) Astr. Ap. Suppl. 47, 1 Heiles, C. (1984) Ap. J. Suppl 55, 585

Heiles, C., Chu., Y.-H., Reynolds, R.J., Yegingil, I., and Troland, T. H. (1980) Ap. J. $242,533$.

IRAS Zodiacal Observation History File (version 3) (1988), as described in the IRAS Explanatory Supplement (1988).

Kerr, F.J., Bowers, P.F., Jackson, P.D., and Kerr, M. (1986) Astr. Ap. Suppl. 66, 373

Lockman, F.J. (1984) Ap. J. 283, 90

Mac Low, M., McCray, R., and Norman, M.L. (1989) Ap. J. 337, 141

Müller, P., Reif, K., and Reich, W. (1987) Astr. Ap. 183, 327

Reynolds, R.J. (1989) Ap. J. Letters 339, L29

Weaver, H., and Williams, D.R.W. (1973) Astr. Ap. Suppl. 8, 1 